\title{
Glokalisierung und Feminisierung: Zur strukturellen Krise von Lohnarbeit im europäischen Raum
}

\author{
Stefanie Hürtgen \\ Fachbereich Geographie und Geologie, Universität Salzburg, Hellbrunnerstraße 34, 5020 Salzburg, Austria \\ Correspondence: Stefanie Hürtgen (stefanie.huertgen@sbg.ac.at)
}

Received: 10 September 2019 - Revised: 14 January 2020 - Accepted: 21 January 2021 - Published: 11 June 2021

Kurzfassung. The article discusses the glocalized socio-spatial form of European production as socially crisisridden. Combining literature from transnational production network theory, critical political economy, labour process theory and feminist geography the article shows that a European production regime has developed which is based on the transnationalization of economic and competitive parameters on the one hand and multiscalar social fragmentation of labour processes on the other. Its very logic is, hence, functional economic integration based on labour's socio-spatial disintegration. The regime pushes for what we can call the feminization of work because it systematically cuts the former, patriarchal and uneven connection between waged work and sociopolitical integration. As feminist debates show, progressive perspectives have to be transnational and multiscalar and they have to include fundamental questions about the concept and status of work in society.

\section{Einleitung}

Die anhaltende ökonomische und soziale europäische Krise steht im Zentrum vieler wirtschafts- und sozialgeographischer Beiträge - auch im deutschsprachigen Diskussionsraum (Klagge, 2009; Belina, 2011; Scheuplein und Wood, 2011; Zeller, 2011; Boeckler und Berndt, 2013; Musil, 2013; Petzold, 2018; Mießner, 2018; Oßenbrügge, 2018). Betrachtet man diese Debatte allerdings näher, ist auffällig, dass die soziale Konstitution von Arbeits- und Produktionsprozessen kaum vorkommt. Schwerpunkte sind die Rolle von Finanzinvestitionen und -spekulationen, Akkumulation durch Enteignung und Inwertsetzung vormalig öffentlicher Güter (beispielsweise im Bereich Wohnen) oder die kommunalen, staatlichen und europäischen Austeritätspolitiken. Dagegen bleiben Beiträge zu krisenhaften Konfigurationen von (Lohn)Arbeit ${ }^{1}$ vereinzelt: Mitunter gibt es Bezüge zur Soziologie (Prekarisierung, Abstiegsgesellschaft, wachsende soziale Ungleichheit u. a.; exemplarisch: Runkel und Everts, 2017), aber diese sind oft wenig theoretisch verdichtet (zu dieser

\footnotetext{
${ }^{1}$ Ich schreibe mitunter von (Lohn-)Arbeit, um auf die Unterschiedlichkeit abhängiger Erwerbsarbeit aufmerksam zu machen; diese kann bekanntermaßen auch informalisierte Formen von (Schein-)Selbständigkeit, Praktika etc. umfassen.
}

Leerstelle Scheuplein, 2015; als Ausnahmen s. Zeller, 2008; Heeg, 2014).

Was in der Diskussion bislang fehlt, das ist eine systematische raumsensible Analyse des Formwandels europäischer (Lohn-)Arbeits- und Produktionsorganisation. Bob Jessop (2012:94) erinnert daran, dass es keine kapitalistische Produktionsweise als solche gibt. Kapitalistische Verwertungskalküle und die Praktiken der Ausbeutung von (Lohn-)Arbeit bestehen in verschiedenen räumlichen und zeitlichen Formationen oder ,sozialen Formen“, wie es die frühe Regulationstheorie in Anschluss an Marx bezeichnet hatte (Aglietta, 1976). In der geographischen Debatte steht eine genauere Betrachtung der gegenwärtigen sozialräumlichen Formen europäischer Produktionsdynamiken bislang aus. Sie ist allerdings dringend, so der Einsatz dieses Beitrages, um über jeweilige akute Zuspitzungen hinaus (,Griechenlandkrise“, „Finanzkrise“, aktuell „Coronakrise“ usw.) die strukturelle Krisenhaftigkeit von (Lohn-)Arbeit in den Blick zu nehmen, das heißt ihre auf Dauer gestellte, normalisierte Abdrängung als Medium sozialer Integration und gesellschaftlicher politischer Partizipation. Diese strukturelle Krisenhaftigkeit ist der gegenwärtigen sozialräumlichen Verfasstheit europäischer Produktionsorganisation inhärent; sie ist ihr nicht äußerlich, wie es Diskussionen zu den „Überflüs- 
sigen“" nahegelegt hatten (Bude, 2002), und wie es neoliberale Diskurse behaupten, die begrifflich die gesellschaftliche Teilhabe von Arbeiter*innen auf eine Teilhabe am Arbeitsmarkt bei weitgehender Ausblendung der Arbeitsbedingungen verschieben.

Im Beitrag analysiere ich die sozialräumliche Form europäischer Produktionsdynamiken und diskutiere ihre Arbeit entsichernde und desintegrierende Dynamik. Ich spreche von einem flexibel transnational-fragmentierenden Produktionsregime, das (Lohn-)Arbeit systematisch von den arbeitsund reproduktionsbezogenen Grundlagen ihrer gesellschaftlichen Integration abtrennt. Es entsteht eine Logik funktionaler (ökonomischer) Integration bei sozialräumlicher Desintegration, die in Anschluss an entsprechende Diskussionen auch als Feminisierung von (Lohn-)Arbeit bezeichnet werden kann.

Die Argumentation wird entlang von drei aufeinander aufbauenden theoretischen, jeweils auch empirisch illustrierten Schritten entwickelt, die zugleich eine Zusammenführung disziplinär oft getrennter Debatten beinhalten:

Im folgenden Kapitel stelle ich das aus der Labour Process Theory stammende Konzept der despotischen bzw. hegemonialen factory regimes von Michael Burawoy (1985) vor. Für die weitere Betrachtung ist seine konzeptionelle Unterscheidung zwischen „Hegemonie“ und „Despotie“ im Betrieb zentral. Zugleich müssen Burawoys Ansätze vergeschlechtlicht und verräumlicht werden (Lee, 1998), erst dann gerät die auch für die weitere Analyse grundlegende doppelte Konstitution von Hegemonie und Despotie innerhalb der Betriebe und Unternehmen, sowie ihre skalare Dynamisierung und ihre Relation zueinander, in den Blick.

In Kapitel 3 ist das zentrale Argument, dass die (vergeschlechtlichte) Ko-Konstruktion von hegemonialen und despotischen (Lohn-)Arbeitsregimen selbst von ihrer fordistischen Form aus dynamisch reskaliert, d. h. glokalisiert wird. Mit Glokalisierung ist nicht, wie in der Debatte vielfach betont, die "Auflösung“ des Nationalstaatlichen zugunsten der lokalen bzw. globalen Scale gemeint (Brenner, 2001; Swyngedouw, 2004; Wissen, 2008; MacKinnon, 2011). Vielmehr bezeichnet der Begriff die sozialräumliche Aufsprengung nationaler und subnationaler Vergesellschaftungszusammenhänge als Bestandteil und Form ihrer Globalisierung bzw. in der vorliegenden Analyse: Europäisierung. Der Nationalstaat bleibt dabei einerseits (z. B. in der Ausgestaltung von Arbeitsgesetzen) zentral, verliert aber insgesamt die Position als dominante Scale (Jessop, 2002). Eben diese Dynamik der Glokalisierung ist grundlegend auch für den Formwandel von (Lohn-)Arbeit und Produktion. Das europäische Produktionsregime ist gekennzeichnet von verwertungslogischer Transnationalisierung der Produktionsorganisation einerseits (Europäisierung von Profit- und Marktkalkülen sowie von Überwachungs- und Kontrolltechniken) und von dynamischer multi-skalarer sozialräumlicher Fragmentierung der Arbeitsprozesse andererseits, und das ist die permanente Aufsprengung und Neuzusammensetzung vergeschlecht- lichter hegemonialer und despotischer Arbeitsregime. Diese glokale Dynamik wird sichtbar, wenn unterschiedliche theoretische Diskussionsstränge zusammengeführt werden - namentlich die der globalen und europäischen Wertschöpfungsketten bzw. Produktionsnetzwerke (Gereffi, 1996; Coe und Yeung, 2015), die Diskussion zur neuen internationalen bzw. regionalen Arbeitsteilung (Fröbel et al., 1977; Massey, 1984) und arbeitssoziologische Debatten zur Fragmentierung und Prekarisierung von (Lohn-)Arbeit.

In Kapitel 4 wird auf die Dimension der staats- und gesellschaftspolitischen, vergeschlechtlichten Marginalisierung von Arbeit abgehoben, und in Kapitel 5 werden Formen der permanenten Restrukturierung und der digitalen Steuerung der Produktion in ihrer Bedeutung für die Krisenhaftigkeit von (Lohn-)Arbeit insgesamt, d. h. sowohl für die despotischen wie die hegemonialen Arbeitsregime, herausgestrichen. Beides, dauerhafte technologische und sozialräumliche „Optimierung“ wie auch der permanente (digitale) konkurrenzielle Vergleich, vervielfachen und dynamisieren nicht nur die sozialräumlichen Fragmentierungen, sondern führen insbesondere auch zu einer sozialpolitischen Destabilisierung der lange Zeit als geschützt angesehenen „Kernbelegschaften“. Dies wird genauer im 6. Kapitel ausgeführt, wo ich - wiederum in Anlehnung an Burawoy - von doppelter Despotisierung spreche. Kapitel 7 führt die Überlegungen mit Blick auf den gesellschaftlichen Stellenwert von Arbeit zusammen.

\section{Die doppelte und politische Konstitution von Despotie und Hegemonie im Betrieb}

Für die Analyse des sozialräumlichen Formwandels europäischer Produktion erweist sich das von Michael Burawoy (1985) entwickelte Konzept der factory regimes als fruchtbarer Ausgangspunkt. Es beinhaltet zwei direkt zusammenhängende Dimensionen kapitalistischer Produktion: Erstens den unmittelbaren Arbeitsprozess (verstanden als Gesamtheit der koordinierten, hierarchisierten und zugleich umkämpften Tätigkeiten und sozialen Beziehungen zur profitorientierten Umwandlung von Rohstoffen in Endprodukte) und zweitens den political apparatus of production, d. h. wirtschafts- und sozialpolitische Institutionen, Normen und Regularien. Gegen (marxistische) ökonomistische Orthodoxien betont Burawoy, dass der Produktionsprozess selbst politisch ist, er ist politics of production: Die staats- und gesellschaftspolitischen Institutionen regulieren und strukturieren die betrieblichen Abläufe und Auseinandersetzungen und werden umgekehrt von dort aktiv mit reproduziert und dabei auch verändert. Zeitlich und geographisch unterscheidet Burawoy vor allem zwei idealtypische factory regimes ${ }^{2}$ : Erstens das despotische, das er als den Prototyp der Marx'schen Analyse bezeichnet (ebd.: 88) und das

\footnotetext{
${ }^{2}$ Burawoy hat international weitere Regime bestimmt, die aber in der Debatte randständig blieben.
} 
von geringer sozialpolitischer Regulierung und deshalb von hoher Marktabhängigkeit der (Lohn-)Arbeiter*innen sowie deren weitgehende Unterordnung unter die kapitalseitigen Produktionsanforderungen gekennzeichnet sei. Das zweite, hegemoniale Regime dagegen versteht Burawoy als kennzeichnend für den fortgeschrittenen (fordistischen) Wohlfahrtskapitalismus. Hier sind die Lohnabhängigen sozial relativ abgesichert und können interessenpolitisch machtvoll auftreten. Entsprechend muss das Management sie einbinden, oder wie Burawoy sagt, ,überzeugen“. Die fortgesetzte prinzipielle Unterordnung der Arbeiter*innen unter kapitalistische Anforderungen im Lohnarbeitsprozess erfolgt deshalb als Aushandlung seiner konkreten zeitlichen, stofflichen und sozialen Ausgestaltung entlang einer dann auch von den Arbeiter*innen getragenen Kompromissbildung ${ }^{3}$. Später ergänzt Burawoy das hegemonial-despotische factory regime, wo aufgrund arbeitspolitischer Partikularisierung die nach wie vor konsensual ausgerichtete Aushandlung zur Konzessionspolitik wird (Burawoy, 1983). Ich konzentriere mich im Folgenden auf die ersten beiden Regime und komme am Ende auf das hegemonial-despotische zurück ${ }^{4}$.

Burawoys relationale Perspektive einer historisch und räumlich spezifischen (gesellschafts-)politischen Verfasstheit kapitalistischer Arbeitsprozesse ist anschlussfähig und instruktiv für die weitere Analyse des europäischen Produktionsregimes. Allerdings sind dafür auch Brüche und Weiterentwicklungen nötig. Für den vorliegenden Zusammenhang muss erstens die mit seinen Konzepten verwobene Entwicklungslogik (von despotischen Regimen in „früheren“ hin zu hegemonialen in ,fortgeschrittenen“ kapitalistischen Gesellschaften) aufgelöst werden (Lee, 1998:160 ff.; Ngai, 2005). Zweitens ist der vor allem staatspolitischen Perspektive Burawoys die Dimension der gesellschafts- und dabei auch alltagspolitischen hinzuzufügen (Massey, 1994). Drit-

\footnotetext{
${ }^{3}$ Es kann hier nicht diskutiert werden, dass Burawoy die sozialpolitische Absicherung von Beschäftigten zugleich als umfassende ideologische Entpolitisierung zeichnet: Statt die Frage nach der Überwindung des Kapitalismus zu stellen, führten die Belegschaften nunmehr nur noch „Spiele“ mit dem Management um kleinteilige Arbeitsverbesserungen (Burawoy, 1979:46 ff.). Der Autor unternimmt hier eine folgenschwere Trennung zwischen angeblich weniger wichtigen „kleinen“ Fragen alltäglichen Lebens und Arbeitens einerseits und den vermeintlich ,großen“ Systemfragen. Dieser traditionsreichen Dualisierung von ,klein“ und „,groß“, von „Lebens-“ und „Systemveränderung“ muss mit Verweis auf feministische Debatten widersprochen werden (Becker-Schmidt, 2017).

${ }^{4}$ Das Konzept der factory regimes ist in der labour geography als Local Labour Control Regime (LLCR) adaptiert worden (Jonas, 1996; Helms und Cumbers, 2006; Pattenden, 2016). Wie allerdings Hasting und MacKinnon (2017) richtig feststellen, kann die Konzeption des Lokalen als relativ kohärent nur ungenügend die auf allen Scales vorangetriebene Fragmentierung von Arbeitsprozessen und Arbeiter*innen fassen. Jüngere Versuche, das LLCR zu multiskalieren, sind wiederum eher (staats-)politisch ausgerichtet (Smith et al., 2018), während ich selbst direkt auf die (Neu-)Konfiguration von Arbeit und Produktion fokussiere.
}

tens schließlich, und hier von besonderer Wichtigkeit, muss das bei Burawoy letztlich mono-skalare, auf die nationalstaatliche Ebene bezogene Konzept des factory regimes sowohl nach innen (in den Betrieb und Nationalstaat hinein) wie nach außen (über nationale und kontinentale Grenzen hinweg) aufgebrochen, d. h. multi-skalarisiert werden.

Die Notwendigkeit der Multiskalarisierung wird mit einem kurzen Blick zurück auf die für Burawoy konzeptionell zentrale fordistische Wohlfahrtsära deutlich. Diese war in der Tat von bis dahin beispielloser arbeitspolitischer rechtlicher Infrastruktur gekennzeichnet, vorangetrieben nicht zuletzt durch soziale Auseinandersetzungen bis hin zur europäischen Streikwelle 1968. Im Ergebnis herrschten im Facharbeitsbereich oftmals ein ruhiger, teilweise selbstbestimmter Arbeitsrhythmus, soziale Beziehungen zum Management, die durchaus auch von Anerkennung der geleisteten Arbeit geprägt waren, und es bestand insbesondere ein wechselseitiges Wissen um soziale und gewerkschaftliche Rechte und ihre mögliche wirkungsvolle Inanspruchnahme (Beaud und Pialoux, 2004). Allerdings stand dieser hegemonialen Position noch ein ganz anderes betriebliches Regime zur Seite, das nicht nur Burawoy ausblendet, sondern das konzeptionell-rückblickend generell oft ,vergessen“ wird (Hürtgen 2015): Der Bereich der sog. ,unqualifizierten“, typischerweise weiblichen und migrantischen Arbeit, paradigmatisch angesiedelt in der taylorisierten Massenfertigung (Castles und Kosack, 2010 [1972]). Die Literatur zeigt hier ein beeindruckendes Maß an Politiken entsubjektivierender Unterordnung und despotisierender Objektivierung (exemplarisch Bednarz-Braun, 1983; Aulenbacher, 1991): Soziale Rechte blieben dem Status willkürlicher persönlicher Genehmigung verhaftet, sexistische und rassistische Übergriffe waren an der Tagesordnung, und interessenpolitische Repräsentation war typischerweise nicht vorhanden. Sozialräumlich waren die zwei Regime hochgradig polarisiert und von einer sozialen Relation der Unnahbarkeit innerhalb der Fabrik gekennzeichnet ${ }^{5}$.

Der kurze Exkurs zeigt die systematische (nicht historische) Notwendigkeit, Vergeschlechtlichung der factory regimes, bzw. wie ich sie im Folgenden bezeichne: Arbeitsregime in die Analyse einzubeziehen. Scale zielt analytisch auf die sozialräumlich ungleiche Reichweite von Normen, Regularien und Institutionen, d. h. auf das Ensemble strukturell ungleicher Repräsentation (Herod, 2011). Dies zieht mit Blick auf (Lohn-)Arbeit eine zweifache Fragestellung nach sich: einmal die nach der Reichweite progressiver Normen und Regularien, beispielsweise sozialer und politischer Rechte, innerhalb einer Hegemonie staatlich abgesicherter Kapitalverwertung - und zweitens die Frage nach dem gesellschafts-

\footnotetext{
${ }^{5}$ Dort, wo während der Welle migrantischer und Frauenstreiks in den 1970er Jahren die Grenzen dieser ,getrennten Welten“ von Fabrikhalle und Büro bzw. Werkstatt überschritten werden konnten, ist dies als große Bereicherung und Ermächtigung geschildert worden (Annie und Werner, 1975; McDowell et al., 2014).
} 
und alltagspolitisch Nicht-Repräsentierten, nach sozialen Formen, Praxen und auch subjektiven Orientierungen der Desintegration, Verdrängung und Abspaltung aus dem als allgemein deklarierten Hegemonialen (Swyngedouw, 1997; Werner et al., 2017). So muss die Analyse von Arbeitsregimen auch mit der hierarchischen kapitalistischen Arbeitsteilung in ihrer grundlegenden sexistischen Aufspaltung in qualifiziert/unqualifiziert, Kern/Rand sowie Gesellschaft/Natur zusammengeführt werden (Jenson, 1998). Die Integration von (Lohn-)Arbeiter*innen-Interessen in hegemonialisierte Normen, Regeln und Institutionen ist entsprechend mit der Frage nach der „dunklen Seite " 6 dieser hegemonialen Konstellation zu verbinden, d. h. mit der Frage nach sexistischer und (hier immer nur am Rande behandelter) rassistischer Despotisierung von Arbeit (McDowell, 1991).

\section{Sozialräumliche Ungleichheit und transnationale Restrukturierung}

Mein Vorschlag ist, die mit dem Ende der fordistischen Wachstumsphase vorangetriebene Transnationalisierung von Produktion als Reskalierung von Arbeitsregimen, genauer: ihrer hierarchisch-vergeschlechtlichten Ko-Konstitution zu begreifen. Die idealtypisch unterschiedenen despotischen und hegemonialen Regime stehen hierbei auf allen Scales von lokal bis europäisch ${ }^{7}$ einerseits in einem polarisierten, sexistisch geframten sozialräumlichen Verhältnis zueinander, das andererseits einer permanenten sozialräumlichen Restrukturierung unterworfen ist, was (Lohn-)Arbeit insgesamt krisenhaft strukturiert.

Für ein Verständnis von transnationaler Produktion als Reskalierung von Arbeitsregimen ist es notwendig, sich die paradigmatischen Veränderungen in der Organisation der kapitalistischen Produktion seit den späten 1960er Jahren in Erinnerung zu rufen. Wie breit diskutiert, spiegeln diese Veränderungen einen verschärften globalen unternehmerischen Wettbewerb, strukturelle „Überakkumulation“, zunehmend disruptive und kurzfristige Markt- und Technologieentwicklungen und nicht zuletzt einen wachsenden Finanzsektor als Katalysator dieser Phänomene wider (Schoenberger, 1988). Die paradigmatische Umbildung in der Produktion besteht darin, dass anstelle der relativ langfristigen fordistischen Fixierung des Kapitals in einer robusten und hochintegrierten Produktion nun die Fähigkeit entscheidend ist, Produktionsund Arbeitsprozesse aufzuspalten, um sie kurzfristig nach den jeweiligen Marktbedingungen und entsprechenden ma-

\footnotetext{
${ }^{6}$ Phelps et al. (2018): „An invitation to the dark side of economic geography“.

${ }^{7}$ An dieser Stelle kann nur erwähnt werden, dass die europäische Ebene ihrerseits Bestandteil einer globalen politökonomischen Dynamik ist (vgl. hierzu Altvater und Mahnkopf, 1997).
}

nagementseitigen „Optimierungsstrategien“ neu zu kombinieren ${ }^{8}$.

Räumlich ist die Aufspaltung und flexible Rekombination der Produktion (und damit: der Arbeitsprozesse) zutiefst multiskalar. Wir sehen dies, wenn wir drei zentrale Debatten über die paradigmatischen Veränderungen in der Produktion und die räumlichen Dimensionen, auf die sie sich jeweils beziehen, zusammenführen. Die erste Diskussion ist die rund um das Konzept der „Neuen Internationalen Arbeitsteilung“ (Fröbel et al., 1977). Diese befasst sich mit Restrukturierungsprozessen auf inter-nationaler Ebene, nämlich mit der Aus- und Verlagerung des eben skizzierten ,unqualifiziert"weiblich-migrantischen Low-end-Bereich der Produktion in den Globalen Süden; der Globale Süden wurde dabei von Anfang an auch als europäischer Süden verstanden, er umfasste auch Länder der europäischen Peripherie wie Spanien oder das damals staatssozialistische Ungarn (ebd.; Frank, 1982). Die zweite räumliche Dimension in der Diskussion veränderter Produktionsstrukturen ist die regionale, paradigmatisch festgemacht am Konzept der ,Spatial Division of Labor“ (Massey, 1984). Auch hier geht es um die sozialräumliche Desintegration der zumeist weiblichen sog. Einfachfertigung, nun allerdings in Richtung der inneren Peripherie der europäischen Zentrumsländer: strukturschwache Regionen mit geringer gewerkschaftlicher Tradition und hoher Arbeitslosigkeit (Lewis, 1983). Die dritte sozialräumliche Dimension ist geprägt von der arbeitssoziologischen ,Fragmenting Work"-Debatte, d. h. Analysen der Fragmentierung, Prekarisierung und Informalisierung von Arbeit in den Betrieben, Produktionshallen und Abteilungen (Marchington et al., 2005; Castel und Dörre, 2009; Flecker, 2009).

In der Zusammenführung dieser drei Debattenstränge wird sichtbar, dass die sog. „Globalisierung von Produktion“ nicht einfach als räumliche Ausdehnung missverstanden werden darf. In der Debatte wird zwar von der Herausbildung „globaler Produktionsnetzwerke“ gesprochen (Gereffi et al., 2005), diese sind aber räumlich besser als transnationale oder eben glokale Produktionsnetzwerke zu bezeichnen. Denn die ,globale“ bzw. ,europäische“ Konfiguration beruht gewissermaßen auf ihrem „Gegenteil“: der multiskalaren sozialräumlichen Aufspaltung von Lohnarbeitsprozessen - zum Zwecke ihrer flexiblen Rekombination über Regionen, Länder und Kontinente hinweg.

Mit Burawoy und den entsprechenden konzeptionellen Erweiterungen können wir diesen Prozess als postfordistische Reskalierung zunächst einmal feminisiert-despotisierter Ar-

\footnotetext{
${ }^{8}$ Organisatorisch kann zwischen interner und externer Segmentierung unterschieden werden; interne Segmente sind firmeneigene, z. B. relativ budgetautarke Produktionsstandorte, Abteilungen oder Kostenstellen sowie kundenspezifische Produktionslinien, Servicecenter oder Arbeitsgruppen. Externe Segmentierung ist der Outsourcing-Prozess, d. h. die Übertragung von Dienstleistungsund Produktionsfunktionen auf flexible und pyramidal orchestrierte Lieferanten (paradigmatisch: Womack et al., 1991). Beide sind hochdynamisch und überschneiden sich.
} 
beitsregime begreifen. Auf allen idealtypisch unterschiedenen Scales (international, regional, lokal-betrieblich) wird ein feminisierter, ,unqualifiziert“-taylorisierter Tätigkeitsbereich geschaffen. Die Montagearbeit der Elektronik- oder Textilfertigung in den Weltmarktfabriken und Exportzonen des Globalen Südens stehen exemplarisch für diesen Prozess. Allerdings finden wir derartige Verhältnisse nicht nur in Asien und Lateinamerika, sondern beispielsweise auch in Südosteuropa oder Polen sowie in den Regionen und Betrieben der europäischen „Zentrumsländer“ (Maciejewska, 2012; Selwyn et al., 2020) ${ }^{9}$. Bei aller Verschiedenheit entsteht auf den unterschiedlichen Scales ein feminisiertes Low-End, das von flexibilisierten Anstellungsformen, repressiven Arbeitsbedingungen, geringer sozialer Absicherung, sehr niedrigen, oft nicht reproduktionssichernden Löhnen und einer de facto weitgehenden Abwesenheit gewerkschaftlicher Repräsentation gekennzeichnet ist:

In den Ländern des europäischen Südens sind Wohlfahrtssysteme bestenfalls ansatzweise entwickelt, die Löhne bewegen sich insbesondere im Blue-Collar-Bereich auch für „Normalbeschäftigte“ und auch in modernen ausländischen Produktionsfabriken verbreitet unterhalb des Reproduktionsnotwendigen (Schipper, 2016; Drahokoupil und Fabo, 2019); hinzu kommt direkte Repression gegen soziale Bewegungen und Gewerkschaften als verbreitetes Element der politics of production. Regional, d. h. mit Bezug auf die inneren Peripherien der europäischen Zentren, treffen wir auf vergleichbare Problematiken, verstärkt durch den ,new local despotism“ (Peck, 2002) post-wohlfahrtsstaatlicher workfarePolitiken, die bestehende soziale Rechte schwächen und (extremen) Niedriglohn fördern. In den letzten Jahren stand hier vor allem die Dienstleistungs- und Logistikbranche im Fokus der Diskussion (Call Center, Paketdienste, Onlinehändler), aber auch die „einfachen“ Montagetätigkeiten klassischer Produktionsbereiche gehören hierzu (Maciejewska, 2012; Hürtgen, 2019). Lokal-betrieblich schließlich zeigt die Debatte zur Fragmentierung und Prekarisierung von Arbeit und Beschäftigung ebenfalls sowohl die wachsende Ungleichheit in den nahräumlichen Arbeitsbedingungen (Hammer und Riisgaard, 2015), wie auch und damit zusammenhängend die materielle und sozial- und gewerkschaftspolitische Vulnerabilisierung breiter Teile der Beschäftigten (Pulignano, 2017; Schmalz und Sommer, 2019).

Glokal entsteht so eine Akkumulationsdynamik, die (Lohn-)Arbeit von den materialen und sozialpolitischen Ressourcen ihrer sozialen Reproduktion abtrennt. Die Senkung der Arbeitskosten bzw. (in marxistischer Terminologie) die Senkung der Kosten des variablen Kapitals (niedrige Löhne, flexible Arbeitszeiten und Beschäftigungsformen, Möglichkeiten kurzfristiger Entlassungen etc.) wird zu ei-

\footnotetext{
${ }^{9}$ Der „,neue Süden“, so formuliert es Fred Scholz (2002) mit Bezug auf Ulrich Beck, ist nicht national oder kontinental zu begreifen, sondern als Ausdruck einer Bewegung der Fragmentierung auf allen sozialräumlichen Scales.
}

nem systematischen Bestandteil des konkurrenziellen Kalküls transnationaler Produktionsorganisation. Sie geht als cost-capability-ratio in die sonstigen Technologie- und Marketingstrategien ein und bleibt auch nicht, wie lange vermutet, auf bestimmte Branchen beschränkt (vgl. Yeung und Coe, 2015; Selwyn, 2019). „Unqualifzierte“ Niedriglohnarbeit wird so - multiskalar - von den Anforderungen integrativer sozialer Reproduktion weitgehend entkoppelt und, worauf ich im nächsten Kapitel eingehe, aus dem Raum (gewerkschafts-)politischer Repräsentation und Öffentlichkeit abgedrängt.

\section{Gesellschaftspolitische Marginalisierung von (Lohn-)Arbeit}

In der Perspektive Burawoys ist diese sozialräumliche Dynamisierung und Reskalierung von feminisiert-despotisierter (Lohn-)Arbeit nicht einfach Resultat von Unternehmensstrategien, sondern nur politisch zu verstehen, als politics of production.

Staatspolitisch ist deshalb ein Blick auf die gegenwärtige Europäisierung und ihren Modus ,neoliberal-negativer Integration" zentral (Altvater und Mahnkopf, 2007:63 ff.). Entscheidend ist, dass spätestens ab den 1980er Jahren explizit auf eine länderübergreifende (europäische) Verallgemeinerung von Sozialstandards verzichtet, vielmehr sozialräumliche Ungleichheit als „Wettbewerb“ stimulierende (und so vermeintlich Prosperität schaffende) Vielfalt konzipiert wird. Während die Regularien für Investitionen, Kapitaltransfer und (innerbetriebliche) Austauschbeziehungen verallgemeinert, d. h. über die Nationalstaaten hinaus hochskaliert (,europäisiert“) werden, gilt für sozialpolitische Belange das Subsidaritätsprinzip: sie bleiben national bzw. werden noch weiter dereguliert (flexibilisiert, lokalisiert usw., vgl. Agnew, 2001). Wie insbesondere Neil Brenner (2004) gezeigt hat, entsteht so eine europäische Formation von glocalizing competition states: Die internationale Aufsprengung sozialer Standards, ihre Deregulierung, ist die - auch vom Nationalstaat moderierte und vorangetriebene - Form, einer länderübergreifend hochskalierten, in den strategischen Kalkülen der transnationalen Konzerne angesiedelten und von diesen sowie den europäisierten Staatsapparaten durchgesetzten Norm der profitorientierten Konkurrenzfähigkeit zu genügen. Diese glokale, konkurrenzielle europäische Staatlichkeit ist selbst Resultat hegemonial durchgesetzter Interessenpolitik transnationaler Kapitalfraktionen (van Apeldoorn, 2002), und sie ermöglicht, reflektiert und befördert die glokalen produktionsseitigen Restrukturierungen (Hürtgen, 2020a).

Alltags- und gesellschaftspolitisch ist die sexistische Konstruktion von despotischer (Lohn-)Arbeit zentral. Diese erfolgt bekanntermaßen als Abdrängung weiblicher Arbeit ins weitgehend Vor-gesellschaftliche, ihre Konstruktion als im „Privaten“ angesiedelte (Natur-)Ressource, als einer Nei- 
gung oder dem Talent ${ }^{10}$ der (weiblichen) Natur entspringend (Phillips und Taylor, 1980; Elson und Pearson, 1981; Wright, 2006). Bestandteil dieser gesellschaftlichen Konstitution von Lohnarbeit als ,,weiblich“" ist ihre Konstruktion als unqualifizierte Arbeit, d. h. als leicht zu beschaffende, sporadisch und zusätzlich einzusetzende und gesellschaftlich wenig bedeutsame Arbeit - und es ist diese (vermeintliche) gesellschaftliche Randständigkeit, die den relativen Ausschluss von sozialintegrativen Arbeitsbedingungen und sozialpolitischen Rechten sowie öffentlich-gesellschaftlicher Repräsentation begründet (Becker-Schmidt, 2017). Im reskalierten glokalen Produktionsregime wird diese Konstruktion - unterschiedlichen Politiken des Gendermainstreamings zum Trotz - nicht nur nicht aufgegeben, sondern als Naturalisierung despotisierter Arbeitsverhältnisse zugespitzt und verallgemeinert: einmal entlang neoliberaler Workfare-Politiken und der Stigmatisierung sozialer Gruppen als letztlich Untüchtige und Unproduktive (Peck, 2002), und ein weiteres Mal entlang jener Narrative, die hochgradig vulnerable und repressive Arbeitsverhältnisse als Resultat von nah- und fernräumlicher Rückständigkeit der Betroffenen interpretieren (Hadjimichalis, 2018). Insgesamt erscheinen so die dunklen Seiten der transnationalisierten Produktion als eine Frage von spezifischen Eigenschaften betroffener Arbeiter*innen. Diese werden als schwach, ,traditionell“" orientiert, ungenügend qualifiziert ${ }^{11}$ und insgesamt den Anforderungen ,moderner“ Arbeit ungenügend gezeichnet, weshalb sie nicht gleichberechtigt an dem partizipieren könnten, was als moderne Ökonomie und demokratische Mitgestaltung verstanden wird (Massey, 2005). Die gesellschaftliche wie betriebliche Produktion vielfacher sozialräumlicher Polarisierung und Desintegration im Hier und Jetzt wird so überführt in eine auf einer ,zivilisatorischen“ Zeitachse angesiedelten Frage ungenügender Fähigkeiten der je ungleich Despotisierten und Subalternisierten - was die sozialen Zusammenhänge und Relationen weitgehend entnennt. Diese Verschiebung despotisierter Arbeitsregime ins Vor-Gesellschaftliche und Randständige ist dabei auch alltagspolitisch wirksam: in Form alltäglicher Stereotypisierungen, in Form einer gewerkschaftspolitischen Fokussierung auf die „Kernbelegschaften“ oder entlang wissenschafts- und alltagspolitischer Debatten, die auf weitere Deregulierung und Fragmentierung orientieren, um wahlweise den betrieblichen, regionalen oder nationalen

\footnotetext{
10 „Talente“ oder „typisch-weibliche Charakteristika“ reichen hier von besonderer Einfühlsamkeit oder ,schlanken Fingern“ bis zur „Freude am Nähen“ (ebd.); dabei wird an entsprechende Konstruktionen weiblicher Arbeiterinnen als ,maidens“ bzw. „Hausfrauen“ angeknüpft (Mies, 1997; Lee, 1998).

11 „Der überwiegende Trend zu höheren Qualifizierungsanforderungen dürfte dazu führen, dass Erwerbspersonen, die zu einer Höherqualifizierung nicht bereit oder fähig sind, kaum mehr geeignete Arbeitsplätze finden [...]. [Nicht wenige, SH] Personen sind [...] mit den Weiterqualifizierungsmaßnahmen überfordert“(Wilke, 1999:245; ähnlich Kern und Schumann, 1984:19 ff.).
}

Standort zu stärken (Blöcker, 2009; Holst und Matuschek, 2013; Hürtgen, 2020b).

\section{Permanente Restrukturierung und konkurrenzielle digitale Steuerung}

Bevor ich am Ende des Beitrages auf den gesellschaftspolitischen Stellenwert von Lohnarbeit zurückkomme, will ich in den folgenden zwei Kapiteln die Betrachtung der glokalen Produktionsorganisation selbst nochmals vertiefen. Denn für die hier verfolgte These einer generellen Krisenhaftigkeit von (Lohn-)Arbeit ist es entscheidend aufzuzeigen, dass die bislang dargelegten naturalisierend-sexistischen Abspaltungen gewissermaßen nicht mehr wirklich funktionieren: die skizzierte glokale sozialräumliche Aufspaltung der kapitalistischen Arbeitsprozesse entlang hegemonialer und despotischer Arbeitsregime ist alles andere als stabil. Stattdessen ist die „Optimierung“ der Produktionsabläufe im Kontext verschärfter unternehmerischer Konkurrenz beschleunigt und als permanente transnationale Restrukturierung auf Dauer gestellt (Siemiatycki, 2012). Die Fragmentierungen und ihre Scales vervielfältigen sich und die sozialräumlichen „Schnittstellen“ und Formen betrieblicher Hegemonie und Despotie werden in Permanenz neu konfiguriert. Diese Dynamik ist insbesondere zentral mit Blick auf die (ehemals) hegemonialen Regime, d. h. die gewerkschaftlich starken, relativ geschützten und öffentlich repräsentierten ,qualifizierten“ Arbeitsbereiche. Diese stellen keine stabilen Inseln dar, werden vielmehr selbst reskaliert und verändern dabei grundlegend ihren Charakter.

Formen und Dynamiken der permanenten Restrukturierung sind vielfältig, an dieser Stelle will ich aber wenigstens drei zentrale identifizieren, um das Ausmaß der auf Dauer gestellten produktionsbezogenen „Optimierung“ und ihre glokale Form sichtbar zu machen.

Eine erste wichtige Dynamik ergibt sich aus dem, was als upgrading von Arbeitsprozessen und Produktionsstätten bekannt ist, d. h. deren technologische und organisatorische Modernisierung. Um die volle Flexibilität im gesamten Netzwerk zu erreichen, werden gerade auch die sog. Low-endBereiche typischerweise relativ schnell modernisiert und in State-of-the-Art-Technologien integriert. In der Tat wird oft übersehen, dass die flexible Fragmentierung und transnationale Rekombination von Arbeitsprozessen auf ihrer weitgehenden technologischen und organisatorischen Standardisierung beruht (Hürtgen et al., 2009; Contractor et al., 2010; Will-Zocholl, 2017 ${ }^{12}$ ). Dieser „Aufwertungsprozess“ beinhaltet auch die Verlagerung von stärker ,qualifizierten“ Arbeitsfunktionen der Koordinierung, Überwachung, Wartung

\footnotetext{
12 ,The relationship between globalisation and standardisation is reciprocal. On the one hand, standardisation enables a global division of labour by dividing work into individual pieces of processing; on the other hand, globalisation enforces standardization to adjust processes across the globe" (ebd.:82).
} 
usw. in nah- und fernräumliche „Niedrigkostenregionen“ (ebd.; Krzywdzynski, 2018). Allerdings führt die ökonomische Modernisierung insgesamt nicht, wie lange Zeit auch wissenschaftlich erwartet, zu einem auch sozialen upgrading der Produktion, d. h. zu einer auch nur relativen sozialen und räumlichen Verallgemeinerung von reproduktionssichernden inklusiven Arbeitsbedingungen. Vielmehr entsteht das, was Alain Lipietz (1997) die „,vierte internationale Arbeitsteilung" nennt und was wiederum ein multiskalares Phänomen darstellt: eine weitgehende Standardisierung von Arbeitsabläufen, Technologien, Normierungen und organisatorischen Strukturen bei nah- und fernräumlicher sozialer Aufspaltung der Beschäftigten.

Die zweite hier zentrale Dynamik betrifft die übergreifende technologische und organisatorische Modernisierung (wesentlich Digitalisierung) auch und gerade in den ehemaligen Zentren. Die Arbeitsforschung zeigt hier, dass fortgesetzte Digitalisierung als Aufspaltung von Arbeitsbereichen in wenige „hochqualifizierte“ und viele „einfache“, dequalifizierte Tätigkeiten erfolgt (Machacek und Hess, 2018). Davon betroffen sind insbesondere die klassischen „Kerne“ der Büro-, Ingenieurs- und White-Collar-Arbeit in den kapitalistischen Zentren, die in den letzten Jahren ihre Verwandlung in digital taylorisierte „Klickwork“-Tätigkeiten der Softwareproduktion und Service-Dienstleistungen erlebten (ebd.). Diese standardisierten Tätigkeiten sind wiederum besonders leicht sozialräumlich umzuorganisieren, beispielsweise in transnational strukturierten shared service centers zu konzentrieren oder umgekehrt wieder neu auf Standorte zu verteilen (aktuell beispielsweise in Richtung Portugal oder Rumänien, Roque, 2018). Dabei ist in der Burawoy'schen Perspektive wichtig: Nicht (moderne) Technik oder die (neotaylorisierten) Tätigkeiten selbst, sondern die vergeschlechtlichte politische Konstitution von (Lohn-)Arbeit ist entscheidend. Neue Formen des (digitalen) Despotismus (Pfeiffer, 2017) entstehen aufgrund einer hierarchischen Konstitution als gesellschaftlich minderwertige Tätigkeit, die als solche vermeintlich keiner starken sozialpolitischen Absicherung bedarf. Dies gilt für die despotisierten Arbeitsregime der Zulieferer- oder Logistikbranche ebenso wie für die „,dequalifizierte“ Klick- und Servicearbeit, letztere nicht selten in Heimarbeit und oft von Frauen getätigt, typischerweise äußerst gering entlohnt, sozialpolitisch nicht abgesichert und in ihrem rechtspolitischen Arbeitnehmer*innenstatus verunklart und vulnerabel (Huws, 2014; Benner, 2015).

Die dritte zentrale Restrukturierungdynamik bezieht sich auf den glokalen Steuerungs-, Kontroll- und Herrschaftsmodus in der Organisation der kapitalistischen Arbeits- und Produktionsprozesse. In der eine Zeitlang vorherrschenden Begeisterung über moderne „flache“ Netzwerkorganisationen ist mitunter die zum Prozess zugehörige Zentralisierung von Kontrolle und Steuerung übersehen worden. Für diese waren wiederum von Beginn an digitale Technologien entscheidend, denn diese Technologie erlaubt eine vergleichende Messung und Zusammenführung der nunmehr nume- risch aufbereiteten Arbeits- und Produktionsergebnisse quer durch die zunehmend komplexen transnationalen Produktionsnetzwerke (Altmann und Deiß, 1998). Die digitale Standardisierung ist gewissermaßen die materiale Manifestation des verwertungslogischen upscaling der Produktionsorganisation. Sie erlaubt über Regionen, Länder und Kontinente hinweg die Kontrolle und Steuerung von ihrerseits flexibelkurzfristig sozialräumlich aufgespaltener (Lohn-)Arbeit.

Digitale Standardisierung wirkt direkt in die Arbeitsprozesse hinein; sie schlägt sich beispielsweise als genormte Technologie und Arbeitsplatzgestaltung nieder, umfasst firmen- oder netzwerkweite vergleichende Optimierungskampagnen und beinhaltet insbesondere transnationale Formate zur permanenten vergleichenden Messung der Arbeitsund Produktionsergebnisse. Hierbei werden die fragmentierten Arbeitsprozesse in Bezug auf Kosten, Effizienz, Flexibilität, Qualität oder Kundenzufriedenheit verglichen bzw. konkurrieren direkt um vorgegebene Zielmargen und den Zuschlag für (weitere) Investitionen gegeneinander, wie beim sog. benchmarking (Greer und Hauptmeier, 2015). Der konkurrenzielle Vergleich reicht bis hinunter zu den einzelnen Produktionslinien, Arbeitsgruppen und individuellen Arbeiter*innen. Er ist dort veralltäglichter Bestandteil des Arbeitsprozesses, beispielsweise über aktuelle SollIst-Visualisierung bereichs- und aufgabenbezogener Arbeitsergebnisse in den Produktionshallen, über regelmäßige „Teambesprechungen“ zu ihrer Evaluation und Optimierung oder auch als digitalisiert-personalisierte Kontrolle einzelner Arbeitsschritte (über Scanner, Beobachtungskameras, digitale Brillen usw.). Hierbei werden nicht nur Parameter wie "selbstverantwortliche“ Kostenersparnis, Output und Qualität standardisiert und vermessen, sondern auch die Bereitschaft zu flexiblen Arbeitseinsätzen, Pünktlichkeit, Anwesenheitsraten oder Sauberkeit am Arbeitsplatz werden numerisch aufbereitet und schlagen sich beispielsweise in der (gekürzten) Auszahlung variabler Lohnbestandteile nieder (Hürtgen et al., 2009; Staab und Geschke, 2020). Digitale Zentralisierung erlaubt, in einem Wort, die Durchsetzung einer transnationalen konkurrenziellen Steuerung, einer veralltäglichten, permanenten Optimierung durch konkurrenziellen Vergleich. Die competitive relations zwischen den vielfach und multi-skalar aufgespaltenen (Lohn-)Arbeitsprozessen und Arbeiter*innen erhalten so eine manifeste hierarchische unternehmenspolitische Form.

\section{Die doppelte Konstitution von Despotie}

Wir sehen an diesen Dynamiken, dass die glokale Aufspaltung in hegemoniale und despotisierte Arbeitsregime nicht stabil ist, vielmehr erleben auch ehemals ,starke“ hegemoniale Regime eine (andauernde) Transformation. Burawoy selbst hat dies früh und hellsichtig wahrgenommen und mit Blick auf die gewerkschaftspolitisch nach wie vor starken Belegschaftsgruppen die Herausbildung eines hegemonial- 
despotischen Regimes analysiert: ,[I]]n all advanced capitalist societies hegemonic regimes are developing a despotic face“ (Burawoy, 1983:603). Anders gesagt: Hegemoniale Regime werden selbst despotisch.

Dieser neue hegemoniale Despotismus ist nicht mit dem direkt feminisiert-despotischen gleichzusetzen. Das institutionelle Repräsentationssystem ist intakt, die Beschäftigten und ihre Gewerkschaften sind nach wie vor in öffentliche Verhandlungen und Kooperationen eingebunden. Despotie entsteht hier nicht aufgrund einer unmittelbaren Negation der Reproduktionsinteressen der Beschäftigten.

Das Despotische entsteht vielmehr in dem, was Burawoy als ,tying of the interests of workers to the fortunes of their employers" (ebd.:602 f.) beschreibt, und was direkt zur glokalen Logik gegenwärtiger Produktionsorganisation zurückführt: Die fortgesetzte gewerkschaftliche Repräsentation und Verhandlung erfolgt unter der (vermeintlich) unhinterfragbaren Prämisse, die Konkurrenzfähigkeit des jeweiligen Unternehmens, Betriebes, Standorts, Büros usw. nicht zu gefährden, vielmehr (permanent) optimieren zu müssen. Das verallgemeinerte, hochskalierte, vermeintlich unangreifbare Verwertungskalkül ist das hegemonial durchgesetzte Erfordernis, in welches sich gewerkschaftliche Forderungen, auf allen Scales, einschreiben (müssen). Gewerkschaftliche Kooperation und Mitsprache wird fortgesetzt, entwickelt sich aber strukturell zum concession bargaining, d. h. zu einem Aushandlungsprozess über die möglichst soziale Ausgestaltung der zu erhöhenden Konkurrenzfähigkeit, mit den sozialen Standards als flexibler Ressource. Lohnarbeiter*innen werden so interessenpolitisch partikularisiert und geschwächt und die konkreten Arbeitsbedingungen grundlegend verschlechtert (vgl. Hudson und Sadler, 1986).

In der Tat zeigt die Arbeitsforschung eindrücklich, dass sich auch die ehemals fordistisch-hegemonialen „Kernbelegschaften" unterhalb der formal fortexistierenden Normalität in einem tiefgreifenden Krisenmodus befinden (Sweeny und Holmes, 2013; Warren, 2019). Das downscaling arbeitspolitischer Kompromissbildung zur Verfasstheit von Arbeit bei gleichzeitigem upscaling ihrer unternehmerischprofitlogischen Vermessung kann auch die ehemals starken, hegemonialen „Kerne“ nicht mehr stabilisieren: Die Zerklüftung tariflicher und sozialer Standards schreitet fort, soziale Zugeständnisse sind häufig, Niedriglohn breitet sich auch für „normal Beschäftigte“ aus, vermeintlich befristete „Extra-Anstrengungen“, d. h. Arbeitsintensivierungen und extensivierungen, werden in immer neuen Kampagnen und Zielmargen zur Regel, ohne dass sie die mehr oder weniger dauerhafte Bedrohung durch Schließung, Verlagerung, Kundenverlust usw. perspektivisch anhaltend zurückdrängen könnten (Detje et al., 2011; Hürtgen und Voswinkel, 2014; Dunkel und Kratzer, 2016). Hinzu kommen kostenkalkuliert knappe Personalressourcen und permanente organisatorische Umstellungen, die auch für die HegemonialDespotisierten zu Entgrenzung und Erschöpfung führen, so dass gesellschaftlich „,normale“ soziale Reproduktion und (gewerkschafts-)politische Teilhabe verbreitet nicht mehr gegeben sind (ebd.; Paugam, 2008; Schröder und Urban, 2012). Zugleich treibt das concession bargaining der hegemonialdespotisierten Belegschaften die sozialräumliche Fragmentierung selbst mit voran, denn die Zugeständnisse für den „Standorterhalt“", den „Produktionszuschlag“ usw. beinhalten insbesondere auch die immer kleinteiligere Aufspaltung der Belegschaften selbst, d. h. die Akzeptanz von weiteren Aus- und Verlagerungen und weiteren Formen prekärer Beschäftigung zur Kostensenkung und Steigerung von Flexibilität.

Kurz: Wir haben es mittlerweile mit einer doppelten, sich überschneidenden glokalen Form der Despotisierung zu tun: der despotisch-hegemonialen und der feminisiertsubalternisierten Form. Während sich in der feminisiertsubalternisierten Form und den hier typischen unmittelbar repressiven Arbeitsbedingungen (sowie deren nah- und fernräumlicher gesellschaftlicher Abspaltung) klassische sexistische und neue neoliberal-autoritäre Politiken verbinden, erleben auch die vermeintlich integrierten Regime zwar keine institutionell diskursive, wohl aber eine soziale Abkopplung ihrer (Lohn-)Arbeit von sozialintegrativen und politischpartizipativen Bedingungen. Auch hier variieren die Formen, sie reichen von Erschöpfung und Entgrenzung bis zu weithin normalisierten Löhnen unterhalb des normal Reproduktionsnotwendigen.

Beide despotische Regime sind nicht nur produktionsseitig, sondern stets auch arbeits- und gesellschaftspolitisch verfasst. Hier zeigt sich in Bezug auf die hegemoniale Despotie (wiederum auf allen sozialräumlichen Scales inklusive der europäischen), dass die fortgesetzte Einbindung von Gewerkschaften in partikularisierende Konkurrenzimperative das downscaling sozialer Rechte allenfalls moderieren, nicht aber verhindern kann (Bieling und Schulten, 2001). In Bezug auf die repressiv-feminisierte Despotie wird von den Nationalstaaten und der EU die Prämisse von workfare und employability vorangetriebenen, d. h. die unternehmerische Anwendungsperspektive von Arbeitskraft zur neuen Grundlage von Sozialpolitik erklärt (Peck und Theodore, 2015). Beide Despotisierungs-Dynamiken überkreuzen sich und fließen im betrieblichen wie gesellschaftlichen downscaling sozialer Rechte und der stets auf neue Weise ungleichen sozial- und arbeitspolitischen Fragmentierung von (Lohn)Arbeiter*innen zusammen.

\section{Feminisierung und Krise im transnationalen Raum der (Lohn-)Arbeit}

Ausgangspunkt dieses Beitrages war die These, dass eine raumsensible Auseinandersetzung mit der ökonomischen und sozialen europäischen Gegenwartskrise auch auf den Formwandel kapitalistischer Arbeits- und Produktionsorganisation fokussieren muss. Ich habe dann über mehrere Argumentationsschritte die gegenwärtige glokale sozialräum- 
liche Form europäischer Produktionsorganisation dargelegt und als flexibel transnational- fragmentierendes Produktionsregime qualifiziert. Ihr Kern ist das upscaling profitlogischer Vermessung, Kontrolle und Steuerung der Lohnarbeitsprozesse, während die reproduktiven Dimensionen von (Lohn)Arbeit multi-skalar fragmentiert und in immer neuen sozialräumlichen Konstellationen konkurrenziell gegeneinandergestellt werden. Die soziale Krise der Arbeit ist dieser glokalen Form als Dynamik funktionaler Integration bei sozialräumlicher Desintegration inhärent; die dynamische desintegrative Aufsprengung und konkurrenzielle Relationierung von Arbeitsprozessen und Arbeitsbedingungen treibt ihre betriebs- wie gesellschaftspolitische Despotisierung voran. Dabei erleben auch die hegemonial-despotisierten Regime eine auf Dauer gestellte Krisen- und Ausnahmesituation, die sie nur relativ von den hochgradig entsicherten Beschäftigten und subaltern-despotisierten Arbeiter*innen unterscheidet.

Ich will im Ausblick des Beitrages auf den eingangs angesprochenen Zusammenhang der vergeschlechtlichten Konstruktion von Hegemonie und Despotie zurückkommen und damit auf Verschiebungen des gesellschaftlichen Stellenwerts von (Lohn-)Arbeit insgesamt.

Für den fordistischen Wohlfahrtskapitalismus hat Robert Castel (2000) ausführlich gezeigt, wie der Ausbau sozialpolitischer Rechte und Infrastrukturen auf der Grundlage einer inhaltichen Neubestimmung von (Lohn-)Arbeit erfolgt. Diese wandelt sich vom einzeln $\mathrm{zu}$ erledigenden und privat-zufällig zu entlohnenden Tagwerk hin zu einer gesellschaftlich-allgemeinen und also öffentlich (sozialrechtlich) auszugestaltenden Angelegenheit (ebd. ${ }^{13}$; Linhart, 2008; Hürtgen, 2017). Demgegenüber bleibt, wie diskutiert, feminisiert-despotisierte (Lohn-)Arbeit auch im Zuge der postfordistischen Reskalierung nicht nur sozialpolitisch, sondern auch gesellschaftspolitisch eine randständige, ja: vorgesellschaftliche Größe.

Wenn nun aber die sexistischen Abspaltungen nicht mehr solide stabilisiert werden können, die Grenzen zwischen betrieblicher Hegemonie und Despotie sozialräumlich immer neu gezogen und dabei unscharf werden und sich die Despotisierung in die (ehemals) hegemonialen Positionen hinein verallgemeinert - dann wird auch der vormals gesellschaftliche Stellenwert hegemonialer (Lohn-)Arbeit angegriffen. Mit Blick auf entsprechende wirtschaftsgeographische Diskussionen (Phillips und Taylor, 1980; McDowell, 1991; Trauger und Fluri, 2019) kann die hier diskutierte doppelte Despotisierung von Arbeit entsprechend als ihre Feminisierung verstanden werden. Der vormals bestehende (wenngleich patriarchal begrenzte) Zusammenhang zwischen (Lohn-)Arbeitsverausgabung und politisch-

\footnotetext{
13 „Der Arbeitsunfall ist beispielsweise nicht einfach ein Unglücksfall, der einem Arbeiter zustößt. Er ist auch eine gesellschaftliche Tatsache, hinsichtlich derer die Vertreter des Allgemeininteresses sich fragen müssen, ob sie hinnehmbar ist, und wenn ja zu welchen Kosten und in welcher Form“ (Castel, 2011:262).
}

gesellschaftlicher Partizipation wird nun generell in Frage gestellt und auseinandergerissen. Unterhalb der fortbestehenden institutionalisierten Normalität verliert Arbeit ihren Status als (zuvor patriarchal ungleich strukturiertes) Medium sozialer Integration $u n d$ gesellschaftlicher Bedeutsamkeit ${ }^{14}$. Stattdessen wird sie privatisiert, d. h. in die Dimension eines vereinzelt zu erledigenden, in seiner,,employability“ quantifiziert vermessenen Jobs gedrängt (Pulignano, 2017). Ob und wie Jobs eine leiblich-soziale Reproduktion von Lebenskraft (Kerstin Jürgens) erlauben und wie (Lohn-)Arbeit gestaltet ist, erscheint immer weniger als öffentlich-allgemeine, wird vielmehr zur Privatangelegenheit jedes/r Einzelnen. Dies ist nicht nur dort der Fall, wo Löhne die normal-soziale Reproduktion nicht (mehr) sichern, sondern auch dort, wo Dauerkrise und ,schlanke“ (Personal-)Ressourcen Erschöpfung, ,Stress“ und den Rückzug auf pure ,private“ Erholung produzieren und kollegiale Kommunikation und kritischeingreifende Mitsprache mindestens deutlich erschweren.

Zusammen mit dieser Abdrängung von (Lohn-)Arbeit als „öffentliches Gut“ (Castel) verschärft sich ihre ebenfalls aus der Konstitution von ,Weiblichkeit“ bereits bekannte diskurspolitische Naturalisierung. Hier gibt es einmal ein romantisierendes und zugleich individualisierendes Narrativ, das besonders auf modern-digitalisierte Tätigkeiten zielt und die Arbeit als das Ausleben besonderer intrinsischer Neigungen und Talente oder einer nicht weiter zu begründenden Lust an Spiel und Spaß beschreibt (Ferrer-Conill, 2018), vergleichbar der diskursiven Konstruktion weiblicher Arbeit in der Textilindustrie als vermeintliche ,Freude am Nähen“" (s. o.). Eine der Konsequenzen ist erneut, dass die so als individuelle Selbst-Verwirklichung atomisiert konstruierte (Lohn-)Arbeit (vermeintlich) auch nur als Zuverdienst entlohnt werden muss (Spamgirl, 2015). Zum zweiten aber wird die Naturalisierung von Arbeit in ihrer repressiven Gestalt entlang des glokalen europäischen Workfare-Regimes radikalisiert und verallgemeinert. Die vermeintlich zurückbleibenden oder defizitär veranlagten Randgruppen sind nun oftmals auch als unwillens und undiszipliniert gezeichnet, was in dieser Logik entsprechende staatliche Maßnahmen gegenüber den offenbar beschränkten Individuen erforderlich macht. Die Ansiedlung feminisierter (Lohn-)Arbeit im VorGesellschaftlichen begegnet uns hier wieder als die umfassende repressive Vereigenschaftung sozialer Gruppen, nämlich als in Bezug auf ihre Arbeitswilligkeit andersartig und das Ökonomische potenziell gefährdend.

Diese Naturalisierung bzw. Kulturalisierung des Sozialen ist bekanntermaßen selbst Bestandteil einer neoliberalen ideologischen Formierung, die massive Verarmung, Desinte-

\footnotetext{
${ }^{14}$ Die in der Corona-Krise entstandene Debatte zur „Systemrelevanz" (reproduktiver) Arbeitsbereiche bekräftigt die Bedeutung des gesellschaftlich-inhaltlichen Status von Arbeit für ihre sozialrechtliche Absicherung, kann aber als Gegenstand nicht mehr in diesem Beitrag diskutiert werden (vgl. erste Analysen hierzu: Aulenbacher, 2020; Detje und Sauer, 2021:51 ff.).
} 
gration und Entsolidarisierung zum Problem der Betroffenen und ihrer vermeintlich problematischen Verhaltensweisen erklärt (Hall, 1996; Butterwegge und Hentges, 2008). Die despotische Hegemonie des partikularen Konkurrenzvorteils ist in anderen Worten auch eine Hegemonie ihrer Deutung als naturalisierte, oft direkt sozialdarwinistische Vereigenschaftung der ungleich Subalternisierten und Despotisierten. Auch in den Betrieben werden die hierarchisch-konkurrenziellen sozialen Relationen vielfach als sexistische, xenophobe, rassistische usw. praktiziert. Nicht von ungefähr wird bereits lange vor der gegenwärtigen Debatte um rechte Einstellungen von Beschäftigten auf das repressiv-naturalisierende framing der sich glokal vervielfältigenden dark sides ökonomischer Entwicklung in den betrieblichen Alltagsdiskursen und -praktiken verwiesen (Zoll, 1984; Zeuner et al., 2007; Stöss, 2017; Sauer et al., 2018; Dörre, 2019; Hürtgen, 2014, 2020b).

Eine kritische Perspektive, die sexistische, xenophobe usw. Einstellungen zurückweist und sich des eigenen demokratischen Wertekanons versichert, bleibt aber ungenügend, wenn nicht zugleich die krisenhaft-desintegrierenden Strukturlogiken aktueller Vergesellschaftung selbst thematisiert werden (Räthzel, 2002) - im vorliegenden Fall die systematisch fragmentierende und desintegrierende Logik transnationaler europäischer Produktionsorganisation. Hierzu gehört auch eine Kritik an gewerkschaftlichen Positionierungen, die sich insgesamt innerhalb der Hegemonie des partikularen Konkurrenzvorteils und entsprechender Fragmentierungslogiken bewegen - sei es, wenn sie betrieblich als ,fraktale Organisationen“ agieren und deregulierende Standortpolitik forcieren, sei es, wenn sie europäische Tarifkoordinationen unterlaufen oder einer herrschend-xenophoben Stimmungsmache gegen ,faule“ Griechen kaum entschlossen entgegentreten (Bispinck und Schulten, 2001; Cumbers et al., 2008; Dörre, 2011; Hadjimichalis, 2018). In der Tat wäre mit Blick auf Gewerkschaften das Kritikpotenzial des Burawoy'schen Begriffs der hegemonialen Despotie (wieder) neu zu entdecken und angesichts der realen Entwicklungen noch über diesen Beitrag hinaus auszuloten.

Das Projekt einer Kritik der Hegemonie des partikularen sozialräumlichen Konkurrenzvorteils kann allerdings, wie dieser Beitrag zeigt, nicht erfolgen, ohne die Frage nach dem Stellenwert von Arbeit und ihrer gesellschaftlichen Gestaltung in den Mittelpunkt zu stellen, in notwendig multiskalarer transnationaler Perspektive. Das schließt Auseinandersetzungen um eine Neubestimmung des gesellschaftlichen Arbeitsbegriffs - nun in einem umfassenden Sinne ein. Eine fundierte kritische political economy of scale hätte diese Aufgabe.

Datenverfügbarkeit. Für diesen Artikel wurden keine Datensätze genutzt.
Interessenkonflikt. Die Autorin erklärt, dass kein Interessenkonflikt besteht.

Danksagung. Ich bedanke mich sehr herzlich bei den drei anonymen Reviewer*innen, die mit ihren sehr konkreten und produktiven Vorschlägen, sowie auch mit ihrer Kritik mein Nachdenken und den Beitrag vorangebracht haben.

Begutachtung. This paper was edited by Benedikt Korf and reviewed by two anonymous referees.

\section{Literatur}

Aglietta, M.: Régulation et Crises de Capitalisme: l'expérience des Etats-Unis, Calman-Lévy, Paris, 1976.

Agnew, J.: How many Europes? The European Union, Eastward Enlargement and Uneven development, Urban Reg. Stud., 8, 29-38, 2001.

Altmann, N. und Deiß, M.: Productivity by Systemic Rationalization: Good Work - Bad Work - No Work?, Econ. Indust. Democr., 19, 137-159, 1998.

Altvater, E. und Mahnkopf, B.: Grenzen der Globalisierung, Westfälisches Dampfboot, Münster, 1997.

Altvater, E. und Mahnkopf, B.: Konkurrenz für das Empire. Die Zukunft der Europäischen Union in der globalisierten Welt, Westfälisches Dampfboot, Münster, 2007.

Annie, C. und Werner, P.: Gegen die linken Phallokraten, Frauen bei Lip, Merve Verlag, Berlin, 1975.

Aulenbacher, B.: Arbeit, Technik, Geschlecht. Industriesoziologische Frauenforschung am Beispiel der Bekleidungsindustrie, Campus, Frankfurt a. M., New York, 1991.

Aulenbacher, B.: COVID-19 - Warnzeichen oder Weckruf? Über die Sorglosigkeit des Kapitalismus und die „Systemrelevanz“ des Sorgens, in: Virenregime, Wie die Coronakrise unsere Welt verändert: Befunde, Analysen, Anregungen, Herausgeber: Schmidinger, T. und Weidenholzer, J., Bahoe Books, Wien, 2020.

Beaud, S. und Pialoux, M.: Die verlorene Zukunft der Arbeiter: Die Peugeot-Werke von Sochaux-Montbéliard, Édition discours, Band 33, UVK Verlagsgesellschaft, Konstanz, 2004.

Becker-Schmidt, R.: Einleitung, in: Pendelbewegungen - Annäherungen an feministische Subjekttheorie, Verlag Barbara Budrich, Opladen, Berlin, Toronto, 9-36, 2017.

Bednarz-Braun, I.: Arbeiterinnen in der Elektroindustrie, DJI Forschungsbericht, München, 1983

Belina, B.: Kapitalistische Raumproduktion und ökonomische Krise, Z. Wirtschaftsgeogr., 55, 239-252, 2011.

Benner, C. (Hrsg.): Crowdwork - Zurück in die Zukunft?: Perspektiven Digitaler Arbeit, Bund-Verlag, Frankfurt a. M., 2015.

Bieling, H.-J. und Schulten, T.: Competitive Restructuring and Industrial Relations within the European Union: Corporatist Involvement and Beyond?, WSI, Düsseldorf, 2001.

Bispinck, R. und Schulten, T.: Zur Kritik der wettbewerbsorientierten Tarifpolitik, in: Interventionen wider den Zeitgeist, Herausgeber: Wagner, H., VSA-Verlag, Hamburg, 209-225, 2001. 
Boeckler, M. und Berndt, C.: Geographies of circulation and exchange III: The great crisis and marketization ,after markets', Prog. Human Geogr., 37, 424-432, 2013.

Blöcker, A.: Mobile Module, in: Beschäftigte in der Globalisierungsfalle?, Herausgeber: Ahlers, E., Kraemer, B., und Ziegler, A., Baden-Baden, 87-106, 2009.

Brenner, N.: The limits to scale? Methodological reflections on scalar structuration, Prog. Human Geogr., 25, 591-614, 2001.

Brenner, N.: Urban governance and the production of new state spaces in western Europe, 1960-2000, Rev. Int. Polit. Econ., 11, 447-488, 2004.

Bude, H.: Das Phänomen der Exklusion, Mittelweg 36, 11, 3-15, 2002.

Burawoy, M.: Manufacturing Consent, University of Chicago Press, Chicago, 1979.

Burawoy, M.: Between the Labour Process and the State: The Changing Face of Factory Regimes Under Advanced Capitalism, Am. Sociol. Rev., 48, 587-605, 1983.

Burawoy, M.: The Politics of Production, Verso, London, 1985.

Butterwegge, C. und Hentges, G. (Hrsg.): Rechtspopulismus, Arbeitswelt und Armut. Befunde aus Deutschland, Österreich und der Schweiz, Verlag Barbara Budrich, Opladen, Farmington Hills, 2008.

Castel, R.: Die Metamorphosen der sozialen Frage, UVK, U-Verl., Konstanz, 2000.

Castel, R.: Die Krise der Arbeit, Hamburger Edition, Hamburg, 2011.

Castel, R. und Dörre, K. (Hrsg.): Prekarität, Abstieg, Ausgrenzung. Die soziale Frage am Beginn des 21. Jahrhunders, Campus, Frankfurt a. M. und New York, 2009.

Castles, S. und Kosack, G.: The function of labour immigration in Western European capitalism, in: Selected Studies in International Migration and Immigrant Incorporation, Herausgeber: Martiniello, M. und Rath, J., Amsterdam University Press, Amsterdam, 21-44, 2010 [1972].

Coe, N. und Yeung, H. W. C.: Global Production Networks, Oxford University Press, Oxford, 2015.

Contractor, F., Kumur, V., und Kundur, S. K.: Reconceptualizing the Firm in a World of Outsourcing and Offshoring, J. Manage. Stud., 47, 1417-1433, 2010.

Cumbers, A., Nativel, C., und Routledge, P.: Labour Agency and Union positionalities in global production networks, J. Econ. Geogr., 8, 369-387, 2008.

Detje, R. und Sauer, D.: Corona-Krise im Betrieb Empirische: Erfahrungen aus Industrie und Dienstleistungen, VSA Verlag, Hamburg, 2021.

Detje, R., Menz, W., Nies, S., und Sauer, D.: Krise ohne Konflikt? Interessen und Handlungsorientierungen im Betrieb, VSA Verlag, Hamburg, 2011.

Dörre, K.: Funktionswandel von Gewerkschaften. Von der intermediären zur fraktalen Organisation, in: Gewerkschaftliche Modernisierung, Herausgeber: Haipeter, T. und Dörre, K., VS Verlag für Sozialwissenschaften, Wiesbaden, 267-298, 2011.

Dörre, K.: Marsch durch die Betriebe? Rechtspopulistische Orientierungen in der Arbeitswelt, Indes, 7, 124-135, 2019.

Drahokoupil, J. und Fabo, B.: Outsourcing, Offshoring and the Deconstruction of Employment, in: The deconstruction of employment as political question, Herausgeber: Serrano, A. und Jepsen, M., Palgrave, Basingstoke, 33-61, 2019.
Dunkel, W., und Kratzer, N.: Zeit- und Leistungsdruck bei Wissensund Interaktionsarbeit. Neue Steuerungsformen und subjektive Praxis, Nomos, Baden Baden, 2016.

Elson, D. und Pearson, R.: Nimble Fingers Make Cheap workers: An Analysis of Women's Employment in the Third World Export Manufacturing, Feminist Rev., 7, 87-107, 1981.

Ferrer-Conill, R.: Playbour and the Gamification of Work, in: Technologies of Labour and the Politics of Contradiction, Herausgeber: Bilić, P., Primorac, J., und Valtýsson, B., Springer,, London, 193-210, 2018.

Flecker, J.: Outsourcing, Spatial Relocation and the Fragmentation of Employment, Competit. Change, 13, 251-266, 2009.

Frank, A. G.: Die gegenwärtige Krise und Perspektiven des Weltsystems, in: Perspektiven des Weltsystems, Herausgeber: Blaschke, J., Campus, Frankfurt a. M., 230-255, 1982.

Fröbel, F., Heinrichs, J., und Kreye, O.: Die neue internationale Arbeitsteilung, Rowohlt, Reinbek, 1977.

Gereffi, G.: Global Commodity Chains, Competit. Change, 1, $427-$ 439, 1996.

Gereffi, G., Humphrey, J., und Sturgeon T.: The governance of global value chains, Rev. Int. Polit. Econ., 12, 78-104, 2005.

Greer, I. und Hauptmeier, M.: Marketization and Social Dumping: Management whipsawing in Europe's automotive industry, in: Market Expansion and Social Dumping in Europe, Herausgeber: Bernaciak, M., Routledge, London, 125-139, 2015.

Hadjimichalis, C.: Crisis Spaces: Structures, struggles and solidarity in Southern Europe, Routledge, London, 2018.

Hall, S.: Race, Articulation and Societies Structured in Dominance, in: Black British Cultural Studies, Herausgeber: Baker, H. A., Diawara, M., und Lindeborg, R., University of Chicago Press, Chicago, IL, 16-60, 1996.

Hammer, N. und Riisgaard, L.: Labour and Segmentation in Value Chains, in: Putting Labour in its Place, edited by: Newsome, K., Taylor, P., Bair, J., and Rainnie, A., Palgrave Macmillan, Basingstoke, 83-99, 2015.

Hasting, T. und MacKinnon, D.: Re-embedding agency at the workplace scale, Environ. Plan. A, 49, 104-120, 2017.

Heeg, S.: The erosion of corporatism?, Eur. Urban Reg. Stud., 21, 146-160, 2014.

Helms, G. und Cumbers, A.: Regulating the new urban poor: Local labour market control in an old industrial city, Space Polity, 10, 67-86, 2006.

Herod, A.: Scale, Routledge, London, New York, 2011.

Hudson, R. und Sadler, D.: Contesting works closures in Western Europe's old industrial regions. Defending place or betrying class?, in: Production, Work and Territory, Herausgeber: Scott, A. und Storper, M., Harper Collins, London, 172-194, 1986.

Hürtgen, S.: Labour as a transnational actor, and labour's national und cultural diversity as an important frame of today's transnationality, Capital Class, 38, 211-238, https://doi.org/10.1177/0309816813514212, 2014.

Hürtgen, S.: Qualifizierung und Polarisierung im transnationalen Raum der Lohnarbeit, in: Die zeitgemäße Arbeitskraft, Herausgeber: Atzmüller, R., Hürtgen, S., und Krenn, M., Beltz Juventa, Weinheim, Basel, 27-104, 2015.

Hürtgen, S.: Der subjektive gesellschaftliche Sinnbezug auf die eigene (Lohn-)Arbeit. Grundlage von Ansprüchen auf Gestaltung von Arbeit und Gesellschaft, in: Leistung und Gerechtigkeit? Das umstrittene Versprechen des Kapitalismus, Herausgeber: Aulen- 
bacher, B., Dammayr, M., Dörre, K., Menz, W., Riegraf, B., und Wolf, H., Beltz Juventa, Weinheim, Basel, 210-227, 2017.

Hürtgen, S.: Network-based Mass Production, Transnational NeoTaylorism, and Socio-Spatial Fragmentation in the Global and European IT Industry, Working Paper No. 10, Geographies of Uneven Development, Universität Salzburg, Salzburg, 2019.

Hürtgen, S.: Precarization of work and employment in the light of competitive Europeanization and the fragmented and flexible regime of European Production, Capital Class, https://doi.org/10.1177/0309816819900123, in press, 2020a.

Hürtgen, S.: Labour-Process-Related Racism in Transnational European Production: Fragmenting Work meets Xenophobic Culturalization among Workers, Glob. Labour J., 11, 18 - 33, 2020 b.

Hürtgen, S. und Voswinkel, S.: Nichtnormale Normalität? Anspruchslogiken aus der Arbeitnehmermitte, in: Vol. 164, Edition Sigma, Berlin, 2014.

Hürtgen, S., Lüthje, B., Schumm, W., und Sproll, M.: Von Silicon Valley nach Shenzen. Globale Produktion und Arbeit in der ITIndustrie, VSA-Verl., Hamburg, 2009.

Huws, U.: Labor in a digital economy, NYU Press, New York, 2014.

Jenson, J.: The talents of women, the skills of men: flexible specialiszation and women, in: The transformation of work?, Herausgeber: Wood, S., Unwin Hyman, London, 141-155, 1989.

Jessop, B.: The Political Economy of Scale, in: Globalization, Regionalization and Cross-Border Regions, Herausgeber: Perkmann, M. und Sum, N. L., Palgrave, Basingstoke, 25-49, 2002.

Jessop, B.: The World Market, Variegated Capitalism and the Crisis of European Integration, in: Globalisation and European Integration, Herausgeber: Nousios, P., Overbeek, H., und Tsolakis, A., Routledge, London, New York, 91-111, 2012.

Jonas, A.: Local Labour Control Regimes, Reg. Stud., 30, 323-338, 1996.

Kern, H. und Schumann, M.: Das Ende der Arbeitsteilung?, Rationalisierung in der industriellen Produktion; Bestandsaufnahme, Trendbestimmung, Beck, München, 1984.

Klagge, B.: Finanzmärkte, Unternehmensfinanzierung und die aktuelle Finanzkrise, Z. Wirtschaftsgeogr., 53, 1-13, 2009.

Krzywdzinski, M.: Die Rolle von Niedriglohnperipherien in den globalen Wertschöpfungsketten der Automobilindustrie. Der Fall Mittelosteuropas, Prokla, 48, 523-544, 2018.

Lee, C. K.: Gender and the South China Miracle. Two Worlds of Factory Women, Univ. of California Press, Berkeley, Los Angeles, London, 1998.

Lewis, J.: Women, Work, and regional development, North. Econ. Rev., 7, 10-24, 1983.

Linhart, D.: Travailler sans les Autres, Éditions du Seuil, Paris, 2008.

Lipietz, A.: The Post-Fordist World: Labor Relations, International Hierarchy and Global Ecology, Rev. Int. Polit. Econ., 4, 1-41, 1997.

Machacek, E. und Hess, M.: Whither ,high-tech'-labor? Codification and (de-)skilling in automotive components value chains, Geoforum, 99, 287-295, 2018.

Maciejewska, M.: Exhausted Bodies and precarious products: Women's Work in a Special Economic Zone for the electronics industry in Poland, Work Organis. Labour Globaliz., 6, 94-112, 2012.

MacKinnon, A.: Reconstructing scale: Towards a new scalar politics, Prog. Human Geogr., 35, 21-36, 2011.
Marchington, M., Grimshaw, D., Rubery, J., und Wilmott, H. (Eds.): Fragmenting Work: Blurring Organizational Boundaries and Disordering Hierarchies, Oxford University Press, Oxford, 2005.

Massey, D.: Spatial divisions of labor: social structures and the geography of production, Methuen, New York, 1984.

Massey, D.: Place, Space, Gender, University of Minnesota, Minneapolis, 1994.

Massey, D.: For Space, SAGE, Thousand Oaks CA, 2005.

McDowell, L.: Life without father and Ford: the new gender order of post-Fordism, T. Inst. Brit. Geogr., 16, 400-419, 1991.

McDowell, L., Sundari, A., und Pearson, R.: Striking Narratives: class, gender and ethnicity in the ,Great Grunwick Strike', London, UK, 1976-1978, Women. Hist. Rev., 23, 595-619, 2014.

Mies, M.: Hausfrauisierung, in: Lexikon der Internationalen Politik, Herausgeber: Albrecht, U. und Vogler, H., Oldenburg Verlag, München, Wien, 207-210, 1997.

Mießner, M.: Spatial planning amid crisis. The deepening of neoliberal logic in Germany, Int. Plan. Stud., 25, 52-71, 2018.

Musil, R.: Das regionale Dilemma der Europäischen Union: räumliche Ungleichgewichte in der gegenwärtigen Wirtschaftskrise, Mitt. Österr. Geogr. Gesell., 155, 61-90, 2013.

Ngai, P.: Made in China: Woman Factory Workers in a Global Workplace, Duke University Press, Durham, London, 2005.

Oßenbrügge, J.: „March for Sozialgeographie“? Rechtspopulismus als Zumutung und die regressive Moderne als Herausforderung der Humangeographie, Geogr. Helv., 73, 309-319, https://doi.org/10.5194/gh-73-309-2018, 2018.

Pattenden, J.: Working at the margins of global production networks: local labour control regimes and rural-based labourers in South India, Third World Quart., 37, 1809-1833, 2016.

Paugam, S.: Die elementaren Formen der Armut, HIS Verlag, Hamburg, 2008.

Peck, J.: Political Economies of Scale: Fast Policy, Interscalar Relations, and Neoliberal Workfare, Econ. Geogr., 78, 331-360, 2002.

Peck, J. and Theodore, N.: Fast Policy: Experimental Statecraft at the Thresholds of Neoliberalism, University of Minnesota Press, Minnesota, 2015.

Petzold, T.: Austerity forever? Die Normalisierung der Austerität in der BRD, Verlag Westfälisches Dampfboot, Münster, 2018.

Pfeiffer, S.: „Industrie 4.0“ in the Making. Discourse Patterns and the Rise of Digital Despotism, in: The New Digital Workplace, Herausgeber: Briken, K., Chillas, S., und Krzywdzinski, M., Palgrave, London, 21-41, 2017.

Phelps N., Atienza, M., und Arias, M.: An invitation to the dark side of economic geography, Environ. Plan. A, 50, 236-244, 2018.

Phillips, A. und Taylor, B.: Sex and skill, Feminist Rev., 6, 79-88, 1980.

Pulignano, V.: Precarious Work, Regime of Competition, and the Case of Europe, in: Precarious Work, Herausgeber: Kalleberg, A. und Vallas, S., Emerald Group Publishing, Bingley, 33-60, 2017.

Räthzel, N.: Developments in the Theories of Racism, in: Europe's New Racism, Herausgeber: The Evens Foundation, Berghahn Books, New York, Oxford, 3-26, 2002.

Roque, I.: Call Center Workers Unite! Changing Forms of Organisation and Representation in the Portugese and British Digital Economy, Work Organ. Labour Global., 12, 79-98, 2018. 
Runkel, S. and Everts, J.: Geographien sozialer Krisen/Krisen sozialer Geographien, Geogr. Helv., 72, 475-482, https://doi.org/10.5194/gh-72-475-2017, 2017.

Sauer, D., Stöger, U., Bischoff, J., Detje, R., und Müller, B: Rechtspopulismus und Gewerkschaften, VSA Verlag, Hamburg, 2018.

Scheuplein, C.: Narration der Arbeit in der deutschsprachigen Humangeographie, in: Arbeit als Narration, Herausgeber: Erdbrügge, T., Nagelschmidt, I., und Probst, I., Klartext, Essen, 89-101, 2015.

Scheuplein, C. und Wood, G. (Hrsg.): Nach der Weltwirtschaftskrise: Neuanfänge in der Region?, LIT Verlag, Berlin, 2011.

Schipper, I.: Electronic assembly in Hungary. How labour fails to protect workers, in: Flexible workforces and low profit margins: electronics assembly between Europe and China, Herausgeber: Drahokoupil, J., Andrijasevic, R., und Sacchetto, D., European Trade Union Institute, Brussels, 131-152, 2016.

Schmalz, S. und Sommer, B. (Hrsg.): Confronting Crisis and Precariousness. Organised Labour and Social Unrest in the European Union, Rowman \& Littlefield International, London, 2019.

Schoenberger, E.: From Fordism to Flexible Accumulation, Environ. Plan. D, 6, 245-262, 1988.

Scholz, F.: Die Theorie der fragmentierenden Entwicklung, Geogr. Rundschau, 54, 6-11, 2002.

Schröder, L. und Urban, H. (Hrsg.): Gute Arbeit, Ausgabe 2012, Zeitbombe Arbeitsstress - Befunde, Strategien, Regelungsbedarfe, Bund-Verlag, Frankfurt a. M., 2012.

Selwyn, B.: Poverty Chains and Global Capitalism, Competit. Change, 23, 71-97, 2019.

Selwyn, B., Musiolek, B., und Ijarja, A.: Making a global poverty chain: export footwear production and gendered labor exploitation in Eastern and Central Europe, Rev. Int. Polit. Econ., 27, 377-403, 2020.

Siemiatycki, E.: Forced to concede: permanent restructuring and labor's place in the North American auto industry, Antipode, 44, 453-473, 2012.

Smith, A., Barbu, M., Campling, L., Harrison, J., und Richardson, B.: Labor regimes, global production networks, and European Union trade policy: labor standards and export production in the Moldovan clothing industry, Econ. Geogr., 94, 550-574, 2018.

Spamgirl: Turken als neue Arbeitsform, in: Crowdwork - zurück in die Zukunft?, Herausgeber: Benner, C., Bund-Verlag, Frankfurt a. M., 99-111, 2015.

Staab, P. und Geschke, S.-C.: Ratings als arbeitspolitisches Konfliktfeld. Das Beispiel Zalando, in: Vol. 429, ZDB-ID 2842948-5, Hans-Böckler-Stiftung, Düsseldorf, 2020.
Stöss, R.: Gewerkschaften und Rechtsextremismus in Europa, Friedrich-Ebert-Stiftung, Berlin, 2017.

Sweeny, B. und Holmes, J.: Problematizing Labour's Agency: Rescaling Collective Bargaining in British Columbia Pulp and Paper Mills, Antipode, 45, 218-237, 2013.

Swyngedouw, E.: Neither global nor local: „Glocalization“ and the politics of scale, in: Spaces of Globalization, Herausgeber: Cox, K., Guilford Press, New York, 137-166, 1997.

Swyngedouw, E.: Globalisation or ,Glocalisation? Networks, Territories and Rescaling', Cambridge Rev. Int. Affair., 17, 25-48, 2004.

Trauger, A. und Fluri, J. L.: Engendering Development: Capitalism and Inequality in the Global Economy, Routledge, Abingdon, New York, 2019.

van Apeldoorn, B.: Transnational Capitalism and the Struggle over European Integration, Routledge, London, New York, 2002.

Warren, A.: Labour Geographies of Workplace Restructuring: An Intra-Labour Analysis, Antipode, 51, 681-706, 2019.

Werner, M., Strauss, K., Parker, B., Orzeck, R., Derickson, K., und Bonds, A.: Feminist political economy in geography, Geoforum, 79, 1-4, 2017.

Wilke, G.: Die Zukunft unserer Arbeit, Campus Verlag, Frankfurt a. M., New York, 1999.

Will-Zocholl, M.: Virtual Temptations: Reorganizing Work under Conditions of Digitisation, Virtualization and Informatisation, in: The New Digital Workplace, Herausgeber: Briken, K., Chillas, S., und Krzywdzinski, M., Palgrave, London, 62-86, 2017.

Wissen, M.: Zur räumlichen Dimensionierung sozialer Prozesse, in: Politics of Scale, Herausgeber: Röttger, B. und Heeg, S., Westfälisches Dampfboot, Münster, 8-34, 2008.

Womack, J., Jones, D., und Roos, D.: The Machine that Changed the World, Harper Perennial, New York, 1991.

Wright, M.: Disposable Woman and other Myths of Global Capitalism, Routledge, New York, London, 2006.

Yeung, H. W. C. und Coe, N.: Toward a Dynamic Theory of Global Production Networks, Econ. Geogr., 91, 29-58, 2015.

Zeller, C.: Globalisierung der Arbeit und der Verunsicherung, Geogr. Z., 96, 78-96, 2008.

Zeller, C.: Verschiebungen der Krise im globalen Rentierregime, Z. Wirtschaftsgeogr., 55, 65-83, 2011.

Zeuner, B., Gester, J., Fichter, M., Kreis, J., und Stöss, R.: Gewerkschaften und Rechtsextremismus, Westfälisches Dampfboot, Münster, 2007.

Zoll, R. (Hrsg.): „Die Arbeitslosen, die könnt ich alle erschießen“ Arbeiterbewusstsein in der Wirtschaftskrise, Bund-Verlag, Köln, 1984. 